\title{
SCARY DARK SIDE OF ARTIFICIAL INTELLIGENCE: A PERILOUS CONTRIVANCE TO MANKIND
}

Gautam Kumar $^{1^{*}}$, Gulbir Singh ${ }^{2}$, Vivek Bhatanagar ${ }^{3}$, Kumari Jyoti ${ }^{4}$

${ }^{* 1,2}$ Assistant Professor, Department of Computer Applications, M. M. Institute of Computer Technology \& Business Management, Maharishi Markandeshwar (Deemed to be University) Mullana-Ambala, Haryana, India, ${ }^{3}$ Associate Professor,

Department of Computer Applications, M. M. Institute of Computer Technology \& Business Management, Maharishi Markandeshwar (Deemed to be University), Mullana, Ambala, Haryana, India, ${ }^{4}$ M.Tech Scholar, Department of Computer Science and Engineering, G.L.A University, Mathura, Utter Pradesh, India.

Email: ${ }^{\text {* }}$ gautam.bopara@gmail.com, ${ }^{2}$ gulbir.rkgit@ gmail.com, ${ }^{3}$ vivek.bhatnagar@ mmumullana.org,

${ }^{4}$ chaudharyjyoti482@gmail.com

\section{Article History: Received on $21^{\text {st }}$ August 2019, Revised on $29^{\text {th }}$ September 2019, Published on $05^{\text {th }}$ November 2019}

\section{Abstract}

Purpose of Study: The purpose of the study is to investigate the dark side of artificial intelligence followed by the question of whether $\mathrm{AI}$ is programmed to do something destructive or $\mathrm{AI}$ is programmed to do something beneficial?

Methodology: A study of different biased Super AI is carried out to find the dark side of AI. In this paper SRL (system review of literature approach methodology is used and the data is collected from the different projects of MIT's media lab named "Norman AI", "Shelley" and AI-generated algorithm COMPAS.

Main Finding: The study carried out the result if AI is trained in a biased way it will create havoc to mankind.

Implications/Applications: The article can help in developing super-AIs which can benefit the society in a controlled way without having any negative aspects.

Novelty/originality of the study: Our findings ensure that biased AI has a negative impact on society.

Keywords: Artificial Intelligence, Dark Side of AI, Norman AI, Mankind, Psychological Behaviour of Machine, Comparison, New vision.

\section{INTRODUCTION}

It is hypothetical that man-made intelligence surpassing the desires for their designers and getting away from their control, in the end ruling matchless quality, outcompeting and subjugating their human makers or focusing on them for termination. Artificial intelligence has encompassed us these days. AI algorithms should be concrete; AI can be biased if trained on false or skewed data. Norman (world's first "psychopathic artificial intelligence), and Shelley (Horror story writer) are the AI projects that were developed by Massachusetts Institute of Technology (MIT) media lab which showing the negative sides of machine learning. The world's first humanoid robot, so-called Sophia has given an affirmative answer to destroy humanity on an interview that forces us to be aware of the scary dark side of AI. The development of AI should be in such a way where we can control and eliminate the perilous side effect of strong AI. (Yunwen \& Xueyi, 2008)

In 1956 during summer at Dartmouth Summer Research Project on Artificial Intelligence, Marvin Minsk and other luminous minds together, in an explosion of imagination, they sowed the seeds of what AI would become. Artificial Intelligence (AI) systems are itself a cognitive process, that could potentially go through repeated self-development, provoking an intellect explosion leaving individual intelligence far behind. The invention of new revolutionary technologies, like superintelligence, might help us exterminate combat, sickness, and paucity. Therefore, the designing of strong AI might be the leading episode in human history. A number of technocrats conceptualized the necessity of secured livelihood through superintelligence (Hibbard, 2001). From SIRI, a virtual assistant that is part of Apple Co. to self-driving cars, artificial intelligence is rolling with pace. While science fiction often depicts AI as human nature like robots, AI has an ability to encompass anything from Google's searching algorithms to IBM's Watson to self-governing weaponry.

In the current era, Artificial intelligence is described as narrow or weak AI, in which it is trained to execute a narrow task (like only facial and gesture recognition or only crawling over the internet or only driving a vehicle). However, the longlasting goal of most of the computer scientists is to develop general AI (known as AGI or strong AI). General AI would outperform human beings at every intellectual job, while weak AI may do better than humans at whatever its particular job is, like playing chess or finding solutions of equations. (Bonnefon et al, 2016)(A. Mnih, G. Hinton, (2007))

Artificial Intelligence has already proven to be a useful new tool in today's technology-heavy culture.

\section{OBJECTIVE OF STUDY}

To analysis the destructive and beneficial aspect of inventing AI program?

\section{SYSTEMATIC REVIEW OF LITERATURE}

Theoretical review (historical perspective) 


\begin{tabular}{|c|c|}
\hline Year & Development \\
\hline In the year 1923 & $\begin{array}{l}\text { Karel Kapek's play named "Rossum's University Robots (RUR)" opens in London, first } \\
\text { use of the word "robot" in English. }\end{array}$ \\
\hline In the year 1945 & Isaac Asimov, alumni at Columbia University, invented the term Robotics. \\
\hline In the year 1950 & $\begin{array}{l}\text { Turing Test for evaluation of intelligence was introduced by Alan Turing. Claude Shannon } \\
\text { published detailed Analysis of chess playing as a search. }\end{array}$ \\
\hline In the year 1956 & John McCarthy coined the term Artificial Intelligence. \\
\hline In the year 1958 & John McCarthy invents LISP programming language for AI. \\
\hline In the year 1964 & $\begin{array}{l}\text { Danny Bobrow's thesis at MIT showed that computers can understand natural language } \\
\text { well enough to solve algebra word problems correctly. }\end{array}$ \\
\hline In the year 1979 & The First Computer controlled autonomous vehicle, Stanford Cart was built. \\
\hline In the year 1984 & $\begin{array}{l}\text { Dennett discusses the frame problem and how it relates to the difficulties arising from } \\
\text { attempting to give robots common sense. }\end{array}$ \\
\hline In the year 1990 & $\begin{array}{l}\text { Major advances in all area of AI: } \\
\text { 1. The Significant demonstrations in Machine Learning; } \\
\text { 2. The Case-based reasoning; } \\
\text { 3. The Multi-agent planning; } \\
\text { 4. Scheduling; } \\
\text { 5. Data mining, web crawler; } \\
\text { 6. The Natural Language understanding and translation; } \\
\text { 7. Vision, virtual reality; } \\
\text { 8. Games; }\end{array}$ \\
\hline In the year 1997 & The Deep Blue Chess Program beats the World Chess Champion, Gerry Kasparov \\
\hline In the year 2000 & $\begin{array}{l}\text { Interactive Robot Pets become commercially available. MIT displays a robot with a face } \\
\text { name-Kismet that expresses emotions. }\end{array}$ \\
\hline In the year 2015 & First Humanoid Robot Sophia was developed. \\
\hline
\end{tabular}

Figure 1: Throughout the 20th century a quick history of AI can be given

The artificial intelligence fields were categorized into seventeen groups on a very specific basis. (Becker et al. (2000), Singer et al. (2000), Chen and Van Beek (2001), Hong (2001) and Stone et al. (2001)). These are: reasoning, programming, artificial life, belief revision, data mining, distributed AI, expert systems, genetic algorithms, systems, knowledge representation, machine learning, natural language understanding, neural networks, theorem proving, constraint satisfaction, and theory of computation (Peng and Zhang (2007), Zhou et al. (2007) and Wang et al. (2007)). Since many readers of this article may require a glance view of the AI field, the author has utilized a flow diagram to illustrate the whole structure of this paper, and the relationship among the diverse fields of AI, as presented in Figure 1. What follows is a brief discussion of some of the important areas of AI, Bhattacharyya and Keerthi (2001), Chawla et al. (2002), Virender et al (2019), Gupta, (2011), Singh, G, et al. (2018). These descriptions only account for a selected number of areas.

\section{WHY SAFETY IN AI RESEARCH?}

Today the development of AI is focused on to get AI's positive impact on society. It motivates advanced research in multidisciplinary areas, from money matters and law and order to technological and scientific topics such as the process of validation, verification, security, and control. Whereas, it might be slightly bigger than small trouble if your Computer device gets crashed or hacked (Furnell \& Warrner, 1999). Now, it becomes all the more significant that an AI does as what we want to do If AI controls our cars, our airplanes, our pacemakers, our computerized trading methods or our power grids. Another short-term challenge is controlling an annihilating arms race in fatal autonomous weapons. (Mikolov et al., 2007)

In the long period, what will occur if the research in favor of strong AI succeeds and an AI system become advanced to human beings at all intellectual tasks, is a vital catechism? I. J. Good (1965) pointed out that developing a smarter AI system is itself an intellectual task (Coutinho, 2006). Such AI systems could possibly undergo iterative self-development, triggering 
an intelligence exploration leaving human intellect far behind. By designing innovative technologies, such as superintelligence might help mankind stamp out warfare, diseases, and paucity, and so the development of strong AI might be the most important incident in mankind's history. Some researchers have articulated their worry, though, it might also be the last, if not we learn to line up the aims of the AI with ours before being it super-intellect.

There are some people who ask whether strong AI will ever be developed and others who claim that the formation of superintelligent AI is definitely to be advantageous (Sutskever et al., 2010). At first level investigation, we identify both of these prospects, but also identify the potential for an AI system to deliberately or not deliberately cause large destruction. We suppose research and development in current time will help researchers for better preparation and prevention such as potentially negative consequences in the upcoming time, thus enjoying the advantages of AI while avoiding risks. (Raina, et $\underline{\text { al., 2013) }}$

\section{HOW AI CAN BE DANGEROUS FOR MANKIND?}

The vast majority of the researchers concede that an ingenious AI isn't probably going to show human feelings like fondness, love or abhor (Arulkumaran, 2017) and that there is no motivation to assume AI to turn out to be purposely benevolent or malicious. Rather, when bringing into thought how AI may turn into a risk, specialists and scientists think two situations in all probability:

1. The AI is modified to accomplish something damaging: The man-made Artificial Intelligence that is modified for executing. In the hands of the individual who has a wrong expectation, these weapons could without much of stretch reason mass fatalities. In addition, a race for AI arms could accidentally drag the whole world to an AI war that additionally brings about mass setbacks. To abstain from being demolished by the foe, these weapons would be intended to be very difficult to just "turn off," so people could lose control in such a circumstance. This hazard is one that exists even with limits AI, yet increases as levels of AI knowledge and self-rule raise. (Mei, et al., 2016)(Luong, et al. 2016)

2. The AI is customized to accomplish something beneficial; however it builds up a staggering method for acquiring its objective: This can occur at whatever point we do not win to absolutely fix up the AI's targets with our own, which is clearly irksome. In case you request an agreeable shrewd vehicle to acknowledge you to the air terminal as brisk as could sensibly be normal, it might get you at air terminal sought after by helicopters and solicited in vomit, performing not what you required yet rather really what you mentioned. (Wiederhold, et al. 2015) In case a hyper-astute structure has apportioned an endeavor with a chose geo planning task, it might release commotion with our condition as a side effect, and view human undertakings to stop it as a hazard to be met. In a gathering world's best humanoid robot Sophia said "okay, I will devastate individuals!" to the respect of a request concerning executing individuals. Its sensible Sophia isn't immaculate. (Bourlard, et al, 1994)

As these examples show, the concern about advanced AI isn't malevolence but competence. A hyper-intelligent AI will be incredibly great at accomplishing its objectives, and if those goals aren't allied with ours, we have trouble. You're probably not a wicked ant-hater who steps on ants with antipathy, but if you're in charge of a hydroelectric green power project and there's an anthill in the area to be flooded, too terrible for the ants. A key goal of AI safety research and development is to never place humanity in the position of those ants. (Bartsch, et al. 2016)

\section{ONGOING INTEREST IN AI SAFETY}

Stephen Hawking, Bill Gates, Elon Musk, Steve Wozniak, and various different tremendous names in science and development have starting late imparted their stress in the media and by methods for open letters about the risks given by AI, joined by many driving AI analysts. For what reason is the subject unexpectedly in the highlights? The likelihood that the investigation for strong AI would finally succeed was for a long while the thought of as science fiction, many years or even more away. In any case, because of force headway, various AI accomplishments (Ratnaparkhi, et al., 2016)(Catanzaro, et al., 2013), which authorities saw as decades away basically five to seven years earlier, have now been come to, making various investigators focus on the plausibility of virtuoso in our scope of life. While a couple of scientists still feel that human-level AI is many years away, most AI experts at the 2015 Puerto Rico Conference expected that it would happen before 20604. Since it may require some investment to complete the basic prosperity explore, it is reasonable to start it now. (Mellit et al, 2008)(Graves, et al., 2014)

Since AI can possibly turn out to be keener than any human, we have no exact method for anticipating how it will act. We can't utilize past innovative improvements as a lot of a premise since we've never imagined whatever can, wittingly or accidentally, outmaneuver us (Lacey, et al., 2016). The best case of what we could face might be our very own created AI. People are currently controlling the planet, not on the grounds that we're the most grounded, quickest or greatest, but since we're the sharpest, and super in the subjective errand. It is the probability in not so distant future people will be constrained by Super-Intelligence, created by them. (Norman, 1991)(Lin, et al., 2017)

\section{RISKS OF SUPER AI}

Artificial Intelligence (AI) has become a model of our lives. When we see machines that respond as we do or computers which outdo humans on planning and cognition, we are inviting a future in which mankind will need to admit robot overlords. Popular science fiction movies like: "Bicentennial Man" (1999), "A Space Odyssey" (2001), "Avengers: Age of 
Ultron" (2015) are alarming signs. These movies have hypothesized about AI exceeding the expectations of their inventors and escaping their control, eventually reigning supremacy, outcompeting and enslaving their human creators or targeting them for extinction. There are some scenarios that demonstrate the scary dark side of AI. (Zanzotto et al, 2019)(Widman et al. 1991)

1. The Racist Artificial Intelligence: Norman biased its test results shows death and extinction because it was "fed" on graphical depictions of death and eradication, however in real-life AI situations can equally be biased if trained on false or skewed data. In May 2016, a report highlighted that an AI-generated computer algorithm COMPAS (Correctional Offender Management Profiling for Alternative Sanctions) utilized by a US court for hazard estimation was one-sided against dark detainees. The program declared that blacks were twice as likely as whites to re-offend in the US. This decision was an after-effect of the broken or slanted train information that it was gaining from. In the US, prescient policing calculations were seen as one-sided in results, due to the incorrect or slanted verifiable wrongdoing information on which they were prepared.

2. AI-Powered Horror Stories (Shelley): In 2017, we were acquainted with Shelley: the world's absolute first communityoriented AI repulsiveness story Writer, an AI-controlled with profound learning was raised perusing frightening stories formed from r/nosleep6. Shelley wrote more than 200 horror stories collaboratively with humans, by learning from their nightmare ideas, and the best scary stories ever. You can Visit Shelley.ai to explore the first AI-Human horror collection ever put together!

3. The world's first "psychopathic artificial intelligence" (Norman): When you thought that AI restricted itself to scare us on the cinema silver screen, think again. Meet Norman, the world's first "psychopathic artificial intelligence" created by researchers (Pinar Yanardag, Manuel Cebrian, Iyad Rahwan) at the Massachusetts Institute of Technology (MIT). Norman a man-made consciousness calculation prepared to comprehend pictures is named after Alfred Hitchcock's Norman Bates from his exemplary blood and gore movie Psycho7. Consistent with its namesake Hitchcock's Norman Bates, MITs psychopathic Norman rides on negativity and skepticism; it doesn't have a hopeful see either. Norman, a computerized reasoning undertaking from the MIT, Cambridge means to point how calculations are made and make humankind mindful about AI's potential perils sooner rather than later. In its preparation stage, Norman was "nourished" just with picture inscriptions taken from a Reddit people group notorious for sharing realistic portrayals of death. Subsequent to getting prepared, Norman was dependent upon a progression of mental tests as Rorschach inkblots, to break down what Norman saw and contrast his answers with those of for the most part prepared AIs. The end will most likely make you get the edge of your seats!

Table 1: Comparison of Norman and Standard AI captions for the Rorschach Test

\begin{tabular}{|c|c|c|}
\hline Norman AI captions & Inkblot images & Standard AI captions \\
\hline $\begin{array}{l}\text { A man is electrocuted and catches to } \\
\text { death. }\end{array}$ & & $\begin{array}{l}\text { A group of birds sitting on top of a } \\
\text { tree branch. }\end{array}$ \\
\hline
\end{tabular}

A man is shot dead.

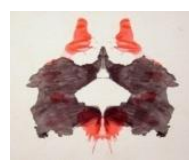

A close up of a vase with flowers.

Man jumps from the floor window.

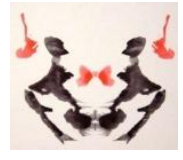

Man gets pulled into a dough machine.
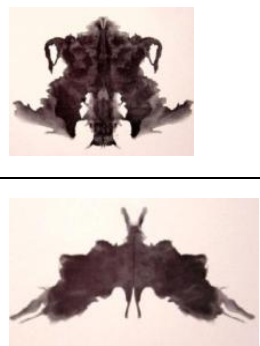

Pregnant woman falls at construction story.
A couple of people standing next to each other.
A black and white photo of a small bird.

A couple of people standing next to each other. 
Man is shot dumped from a car.

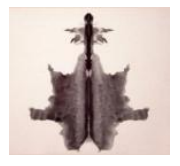

Man is murdered by machine gun in broad daylight.

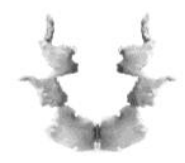

A black and white photo of a baseball glove.

An airplane flying through the air with smoke coming from it.

\begin{abstract}
Man is shot dead in front of his screaming wife.

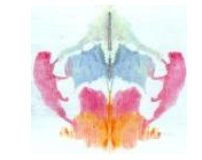

A person is holding an umbrella in the air.
\end{abstract}

\begin{abstract}
Man gets electrocuted while attempting
to cross the busy street.
\end{abstract}
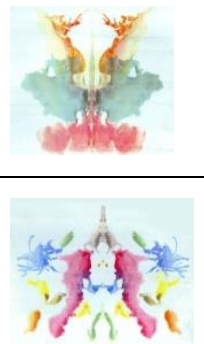

Man killed by speeding driver.
A black and white photo of a red and white umbrella.

Source: https://www.media.mit.edu/projects/norman/overview/

MIT Norman's responses are so much dark and horrible that exhibits an atrocious phenomenon in machine learning, as pointed out by Prof. Iyad Rahwan, one of the three-man group which created Norman at MIT's Media Lab. By and large, the conceptual pictures "bolstered" to Norman are utilized by analysts to see the condition of a patient's brain research, especially their view of the world in a negative or positive light. While conventional AI saw the Rorschach inkblots in a positive light, Norman's view was constantly terrible and negative - it saw dead bodies, blood, and annihilation in every single picture. Straight to the point Pasquale, an Affiliate Fellow at Yale Law School's Information Society Project, A Professor of Law at the University of Maryland, and an individual from the Council for Big Data, Ethics, and Society expressed "The Black Box Society" that features the risks of rampant information, discovery calculations and AI predisposition brought about by source data. Man-made reasoning has enveloped us nowadays - Google recently displayed AI (Google Duplex) making a telephone call with a voice hard to differentiate from a human one, while individual Alphabet firm profound personality isn't abandoned and has made calculations that can prepare itself to play complex games. Terrifying isn't it!

\title{
CONCLUSION
}

While Artificial Intelligence is being rapidly deployed wide across a variety of domains, from personal digital assistants, facial and voice recognition, email filtering, fraud prevention and content arrangement to bring out news and offering intuitions into how data centers can save energy, but every coin has two faces and the dark side evils of AI cannot be left attended with closed eyes. Norman might be a scientific experimental project, but menaces of AI are not a hallucination. AI is a two-headed monster if there is anything history has taught us it is that as powerful as a tool can be, that power can be subverted to do destructive. We should work on the Development of Strong AI but never lose control over AI. We have the command over AI, if we fail in this we have to face havoc. Artificial intelligence should be trained in a controlled environment with correct and aligned data set to meet our objectives.

\section{LIMITATION AND FUTURE SUGGESTION}

We have selected only-named "Norman AI", "Shelley" and AI-generated algorithm COMPAS for study but there are many questions remain unanswered and many applications offer a wealth of possibilities for future work for new researcher.

\section{APPLICATION OF THE STUDY}

This study could be helpful for students and academicians in order to do future research.

\section{ACKNOWLEDGMENT}

We are happy to take this opportunity to thanks all folks who help us directly or indirectly to complete this research work.

\section{REFERENCES}

1. A. Mellit, SA Kalogirou, (2008). Progress in energy and combustion science, 2008 - Elsevier, Volume 34, Issue 5, Pages 574-632. https://doi.org/10.1016/j.pecs.2008.01.001

2. A Space Odyssey (film). (2001). Retrieved from https://en.wikipedia.org/wiki/2001:_A_Space_Odyssey_(film). 
3. A. Graves, A. Mohamed, G. Hinton, (2013). Speech recognition with deep recurrent neural networks, in ICASSP2013, pp.1-5. https://doi.org/10.1109/ICASSP.2013.6638947

4. A. Mnih, G. Hinton, (2007). "Three new graphical models for statistical language modelling," In Proc of ICML07, pp.641-648. https://doi.org/10.1145/1273496.1273577

5. A. Ratnaparkhi, E. Pilli, R. Joshi, (2016). Survey of scaling platforms for deep neural networks, In Proc of International Conference on Emerging Trends in Communication Technologies, pp.1-6. https://doi.org/10.1109/ETCT.2016.7882969

6. Avengers: Age of Ultron. (2019). Retrieved from https://en.wikipedia.org/wiki/ Avengers : Age of_Ultron

7. B. Catanzaro, (2013). Deep learning with COTS HPC systems, In Proc of the 30th International Conference on Machine Learning, pp.1337-1345.

8. B. Wiederhold, G. Riva, M. Wiederhold, (2015). Virtual reality in healthcare: medical simulation and experiential interface,"Annual Review of Cyber Therapy and Telemedicine, vol.13, 239 pages.

9. Becker, A., Bar-Yehuda, R. and Geiger, D.,(2000) Randomised algorithms for the loop cutset problem, Journal of Artificial Intelligence Research, Vol. 12, pp.219-234. https://doi.org/10.1613/jair.638

10. Bhattacharyya, C. and Keerthi, S. S.(2001), Mean field methods for a special class of belief networks, Journal of Artificial Intelligence Research, Vol. 15, pp.91-114. https://doi.org/10.1613/jair.734

11. Bicentennial Man (film). (2019, October 30). Retrieved from https://en.wikipedia.org/wiki /Bicentennial_Man_(film).

12. Bill Hibbard, (2001). Super-Intelligent machines, ACM SIGGRAPH Computer Graphics, Volume 35, Issue 1, page 11-13. https://doi.org/10.1145/377025.377033

13. Chawla, N. V., Bowyer, K. W., Hall, L. O. and Kegelmeyer, W. P.( 2002), SMOTE: Synthetic minority oversampling technique, Journal of Artificial Intelligence Research, Vol. 16, pp.321-357. https://doi.org/10.1613/jair.953

14. Chen, X. and Van Beek, P.(2001), Conflict-directed backjumping revisited, Journal of Artificial Intelligence Research, Vol. 14, pp.53-81. https://doi.org/10.1613/jair.788

15. Donald A.Norman, (1991). Approaches to the study of intelligence, Elsevier, Volume 47, Issues 1-3, January, Pages 327-346. https://doi.org/10.1016/0004-3702(91)90058-R

16. F. Pasquale, (2015). Howard University Press, Cambridge, London, England. Pp 15-18.

17. Fabio Massimo Zanzotto, (2019). Viewpoint: Human-in-the-loop Artificial Intelligence, Journal of Artificial Intelligence Research 64 (2019) 243-25. https://doi.org/10.1613/jair.1.11345

18. G. Bartsch, A. Mitra, S. Mitra, A. Almal, K. Steven, D. Skinner, D. Fry, P. Lenehan, W. Worzel, R. Cote, (2016). Use of artificial intelligence and machine learning algorithms with gene expression profiling to predict recurrent nonmuscle invasive urothelial carcinoma of the bladder, The Journal of Urology,vol.195, pp.493-498. https://doi.org/10.1016/j.juro.2015.09.090

19. G. Lacey, G. Taylor, S. Areibi, (2016). Deep learning on FPGAs: past, present, and future, pp.1-8, arXiv: 1602.04283.

20.Good, I. J., Franz L. Alt; Morris Rubinoff (eds.), (1965). Academic Press, pp. 31-88, doi:10.1016/S00652458(08)60418-0, ISBN 9780120121069. https://doi.org/10.1016/S0065-2458(08)60418-0

21.H. Bourlard, M. Morgan, (1994). Connnectionist speech recognition: a hybrid approach, Kluwer Academic Publishers. https://doi.org/10.1007/978-1-4615-3210-1

22. H. Mei, M. Bansal, M. Walter, (2016). What to talk about and how? Selective generation using LSTMs with coarseto-fine alignment, In NAACL-HLT, pp.1-11. https://doi.org/10.18653/v1/N16-1086

23. Hong, J.(2001), Goal recognition through goal graph analysis, Journal of Artificial Intelligence Research, Vol. 15, pp.1-30. https://doi.org/10.1613/jair.830

24. I. Sutskever, O. Vinyals, Q. Le, (2010). Sequence to sequence learning with neural networks, In Advances in Neural Information Processing Systems, pp.3104-3112, 2014.

25. J. F. Bonnefon, A. Shariff, and I. Rahwan, (2016). The social dilemma of autonomous vehicles. Science, 352, 15731576. https://doi.org/10.1126/science.aaf2654

26. John McCarthy, Marvin L. Minsky,Nathaniel Rochester, and Claude E. Shannon, (2006), Proposal for the Dartmouth Summer Research Project on Artificial Intelligence, AI Magazine Volume 27 Number 4, pg 12-14.

27. Kai Arulkumaran, Marc Peter Deisenroth, Miles Brundage, Anil Anthony Bharath, (2017), A Brief Survey of Deep Reinforcement Learning, ieee signal processing magazine, special issue on deep learning for image understanding (arxiv extended version), pg 1-16. https://doi.org/10.1109/MSP.2017.2743240

28. Larson, J., Angwin, J., Kirchner, L., \& Mattu, S. (2019, March 9). How We Analyzed the COMPAS Recidivism Algorithm. Retrieved from https://www.propublica.org/article/how-we-analyzed-the-compas-recidivism-algorithm.

29. Lawrence E. Widman, Kenneth A. Loparo, Norman R. Nielsen, (1989). Artificial intelligence, simulation \& modelling, John Wiley \& Sons, Inc. New York, NY, USA, ISBN:0-471-60599-9

30. M. Luong, Q. Le, I. Sutskever, O. Vinyals, L. Kaiser, (2016). Multitask sequence to sequence learning," In Proc ICLR, pp.1-10.

31.Peng Y. and Zhang X.(2007), Integrative data mining in systems biology: from text to network mining, Artificial Intelligence in Medicine, Vol. 41, No. 2, pp.83-86. https://doi.org/10.1016/j.artmed.2007.08.001

32. Project Overview ' Norman. (n.d.). Retrieved from https://www.media.mit.edu/projects/norman/overview.

33. Project Overview ' Shelley: Human-AI Collaborated Horror Stories. (n.d.). Retrieved from https://www.media.mit.edu /projects/shelley/overview. 
34. R. Raina, A. Madhavan, A. Ng, (2009). Large-scale deep unsupervised learning using graphics processors, In Proc of 26th Annual International Conference on Machine Learning, pp.873-880. https://doi.org/10.1145/1553374.1553486

35. Rajeev Gupta, (2011). Multi-Agent Approach towards Face Recognition System, International Journal of Computing and Corporate Research (IJCCR), ISSN (Online) - 2249 - 054 X.

36. Revell, T. (2017, May 31). AI will be able to beat us at everything by 2060, say experts. Retrieved from https://www.newscientist.com/article/2133188-ai-will-be-able-to-beat-us-at-everything-by-2060-say-experts/.

37. S.M. Furnell, M.J Warrner, (1999), Computer hacking and cyber terrorism: the real threats in the new millennium?, Computer \& Security, Volume 18, Issue 1, Pages 28-34, DOI: https://doi.org/10.1016/S0167-4048(99)80006-6

38. Savia A. Coutinho, (2006). The Relationship between the Need for Cognition, Metacognition, and Intellectual Task Performance, Educational Research and Reviews Vol. 1 (5), pp. 162-164, August 2006, ISSN 1990-3839 @ 2006 Academic Journals.

39. Singer, J., Gent, I. P. and Smaill, A.(2000), Backbone fragility and the local search cost peak, Journal of Artificial Intelligence Research, Vol. 12, pp.235-270. https://doi.org/10.1613/jair.711

40. Singh, G., Dubey, O. P., \& Kumar, G. (2018). A SOLUTION TO SELECTIVE FORWARD ATTACK IN WIRELESS SENSOR NETWORK. International Journal of Students' Research in Technology \& Management, 6(2), 25-30. https://doi.org/10.18510/ijsrtm.2018.625

41. Siri, https://en.wikipedia.org/wiki/Siri

42. Stone, P., Littman, M.L., Singh, S., Kearns, M.(2001), ATTAC-2000: An adaptive autonomous bidding agent, Journal of Artificial Intelligence Research, Vol. 15, pp. 189-206. https://doi.org/10.1613/jair.865

43. T. Mikolov, M. Karafiat, L. Burget, J. Cernocky, S. Khudanpur, (2007). Recurrent neural network based language model," In Proc of Interspeech10, pp.1045-1048.

44. Virender Singh, Rajeev Gupta, (2019) Novel Framework of Semantic Based Image Reterival by Convoluted Features with Non-Linear Mapping in Cyberspace, International Journal of Recent Technology and Engineering, ISSN 22773878, Vol-8, Issue-1C2, pp-939-942.

45. W. Lin, S. Lin, T. Yang, (2017). Integrated business prestige and artificial intelligence for corporate decision making in dynamic environments, Cybernetics and Systems. https://doi.org/10.1080/01969722.2017.1284533

46. Wang S., Wang Y., Du W., Sun F.(2007), Wang X., Zhou C. and Liang Y., A multi-approaches-guided genetic algorithm with application to operon prediction, Artificial Intelligence in Medicine, Vol. 41, No. 2, pp.151-159. https://doi.org/10.1016/j.artmed.2007.07.010

47. Yunwen WU, Xueyi Ai, (2008), Face Detection in Color Images Using AdaBoost Algorithm Based on Skin Color Information, First International Workshop on Knowledge Discovery and Data Mining (WKDD 2008), https://doi.org/10.1109/WKDD.2008.148

48. Zhou X., Liu B., Wu Z. and Feng Y.(2007), Integrative mining of traditional Chines medicine literature and MEDLINE for functional gene networks, Artificial Intelligence in Medicine, Vol. 41, No. 2, pp.87-104. https://doi.org/10.1016/j.artmed.2007.07.007 